\title{
The Birthday Problem and Zero-Error List Codes
}

\author{
Parham Noorzad, Member, IEEE, Michelle Effros, Fellow, IEEE, \\ Michael Langberg, Senior Member, IEEE, and Victoria Kostina, Member, IEEE
}

\begin{abstract}
A key result of classical information theory states that if the rate of a randomly generated codebook is less than the mutual information between the channel's input and output, then the probability that that codebook has negligible error goes to one as the blocklength goes to infinity. In an attempt to bridge the gap between the probabilistic world of classical information theory and the combinatorial world of zero-error information theory, this work derives necessary and sufficient conditions on the rate so that the probability that a randomly generated codebook operated under list decoding (for any fixed list size) has zero error probability goes to one as the blocklength goes to infinity. Furthermore, this work extends the classical birthday problem to an information-theoretic setting, which results in the definition of a "noisy" counterpart of Rényi entropy, analogous to how mutual information can be considered a noisy counterpart of Shannon entropy.
\end{abstract}

Index Terms-Birthday problem, collision probability, hash function, hypergraph, Körner graph entropy, list decoding, Motzkin-Straus theorem, random coding, Rényi entropy, zeroerror channel capacity.

\section{INTRODUCTION}

Finding channel capacity under a zero-error constraint requires tools and ideas that are fundamentally different from the study of capacity under an asymptotically negligible error constraint; the former relies mostly on graph theory [1], while the latter mainly applies probabilistic arguments [2]. To obtain a better understanding of the relationship between zero-error information theory and classical information theory, we apply probabilistic tools to the study of zero-error channel coding.

The random code construction of Shannon [2] shows that for the discrete memoryless channel $(\mathcal{X}, W(y \mid x), \mathcal{Y})$, a sequence of rate- $R$ codebooks $\left(\mathscr{C}_{n}\right)_{n=1}^{\infty}$, randomly generated ${ }^{1}$ according to distribution $P(x)$, achieves

$$
\lim _{n \rightarrow \infty} \mathbb{E}\left[P_{e}^{(n)}\left(\mathscr{C}_{n}\right)\right]=0,
$$

This paper was presented in part at the 2017 IEEE International Symposium of Information Theory in Aachen.

This material is based upon work supported by the National Science Foundation under Grant Numbers 1321129, 1527524, and 1526771.

P. Noorzad was with the California Institute of Technology, Pasadena, CA 91125 USA. He is now with Qualcomm Technologies, Inc., San Diego, CA 92121 USA (email: parham.n@outlook.com).

M. Effros and V. Kostina are with the California Institute of Technology, Pasadena, CA 91125 USA (emails: effros@caltech.edu, vkostina@ caltech.edu).

M. Langberg is with the State University of New York at Buffalo, Buffalo, NY 14260 USA (email: mikel@buffalo.edu).

${ }^{1}$ By a "randomly generated codebook" we mean a codebook where every symbol of every codeword is generated i.i.d according to some fixed distribution. See, for example, [3 p. 200]. if $R<I(X ; Y)$. In $\sqrt{1}), P_{e}^{(n)}\left(\mathscr{C}_{n}\right)$ is the average probability of error of codebook $\mathscr{C}_{n}$. From Markov's inequality, it follows that $R<I(X ; Y)$ suffices to ensure that

$$
\forall \epsilon \in(0,1): \lim _{n \rightarrow \infty} \operatorname{Pr}\left\{P_{e}^{(n)}\left(\mathscr{C}_{n}\right) \leq \epsilon\right\}=1 .
$$

Our aim is to understand the behavior of randomly generated codebooks when $\epsilon=0$ in (2). Specifically, we seek to find necessary and sufficient conditions on the rate $R$ in terms of the channel $W$ and input distribution $P(x)$ such that the sequence of randomly generated codebooks $\left(\mathscr{C}_{n}\right)_{n=1}^{\infty}$ satisfies

$$
\lim _{n \rightarrow \infty} \operatorname{Pr}\left\{P_{e}^{(n)}\left(\mathscr{C}_{n}\right)=0\right\}=1 .
$$

In other words, our goal is to quantify the performance of randomly generated codebooks under the zero-error constraint. However, we do not limit ourselves to the case where the decoder only has one output. Instead, as in works by Elias [4], [5], we allow the decoder to output a fixed number of messages. This added generality allows us to consider the birthday problem as a special case of our setup, as we see below. We say a codebook corresponds to a "zero-error $L$ list code" if for every message the encoder transmits, the decoder outputs a list of size at most $L$ that contains that message. As with the zero-error list capacity problem [6], the design of a zero-error $L$-list code uses only knowledge of the distinguishability hypergraph of the channel. We discuss hypergraphs and their application to zero-error list codes in Section III We then present our main result in Theorem 4 of Section IV] where we provide upper and lower bounds on the rate of randomly generated zero-error list codes. The proofs of all of our results appear in Section $\mathrm{V}$

The well-known "birthday problem" is a special case of our setup. Specifically, as we next discuss, this special case occurs when the channel $W$ is noiseless, that is, $\mathcal{Y}=\mathcal{X}$, and

$$
\forall(x, y) \in \mathcal{X} \times \mathcal{Y}: W(y \mid x)=\mathbf{1}\{y=x\} .
$$

The classical birthday problem studies the probability that a fixed number of individuals in a population have the same birthday under the assumption that the birthdays are independent and identically distributed (i.i.d.). While this probability has a closed-form expression when the i.i.d. distribution is uniform, no closed-form solution is available in the non-uniform case. Numerical approximations for this probability in the non-uniform case are given in [7]-[9].

We next describe the connection between the birthday problem and our setup above. Consider the problem of channel coding over the noiseless channel defined by (3). Note that 
over this channel, a codebook corresponds to a zero-error $L$ list code if and only if no group of $L+1$ messages are mapped to the same codeword. Associating codewords with birthdays, we obtain an information-theoretic formulation of the birthday problem: Given a randomly generated codebook (which here represents a set of birthdays), what is the probability that some subset of $L+1$ codewords (birthdays) are identical? In Corollary 8, assuming this probability converges to one, we provide the precise asymptotic behavior of the codebook size in terms of the Rényi entropy of order $L+1$.

In the next section, we describe related work.

\section{PRIOR WORK}

Relevant prior work includes the zero-error list decoding literature and the birthday problem literature, both of which we briefly summarize below. For a broader overview of zeroerror information theory, we refer the reader to Körner and Orlitsky [10].

In the zero-error list decoding literature [4], [6], [11], [12] the focus is on constructing codes that achieve the zeroerror list decoding capacity of the channel. In general, this capacity may be different from the highest rate achievable using randomly generated codebooks. As an example, consider the noiseless channel defined by (3). For this channel, the zeroerror capacity, which coincides with zero-error list capacity for list size one, equals $\log |\mathcal{X}|$, while the probability of a rate- $R$ codebook, generated uniformly at random, having zero error on this channel goes to one if and only if

$$
R<\frac{1}{2} \log |\mathcal{X}|
$$

Thus even on a noiseless channel, randomly generated codes only achieve half the capacity. However, it is exactly this distinction with capacity that makes this problem of interest in a wide variety of fields, both within information theory [13], [14] and in areas such as probability theory [9], [15], [16] and cryptography [17] $-[20]$. These latter works are part of the birthday problem literature, which we next describe.

Our work differs with the birthday problem literature mainly in the channels under consideration. Specifically, as we seek to find the maximal rates of randomly generated list codes that have zero error probability over arbitrary channels, our study generalizes that of the birthday problem which, under different asymptotic regimes, addresses the same question with respect to the noiseless channel.

In [13], Rényi states a result in terms of the random "subdivisions" of a set, which we reformulate in Appendix $\mathrm{B}$ in terms of zero-error $L$-list codes over a noiseless channel. In addition to the channel, Rényi's result differs from ours in the asymptotic regime under consideration.

Another related work is [14], where Fujiwara studies a variation of the birthday problem for the case $L=1$ in the setting of quantum information theory. Specifically, Fujiwara determines the maximum growth rate of the cardinality of a sequence of codebooks that satisfy an "asymptotic strong orthogonality" condition.

In probability theory, Arratia, Goldstein, and Gordon [15], [16] apply the Chen-Stein method to approximate the probability of two or more coinciding birthdays for both uniform and non-uniform distributions and also provide error bounds for their approximation. Following these works, it is possible to apply the Chen-Stein method to the birthday problem over arbitrary channels; however, a direct application of the bounds in [15], [16] leads to weaker asymptotic results than those we present here.

The birthday problem also arises in the context of cryptography. For a given hash function, the quantity of interest is the number of hash function evaluations required to find a "collision," where a collision here refers to two inputs that are mapped to the same output ${ }^{2}$ In this context, the default assumption is that the hash function values are uniformly distributed on their alphabet, as this leads to the lowest collision probability [19, p. 192]. However, Bellare and Kohno [17] argue that the uniformity assumption need not hold for real-world hash functions. Thus, it is also important to study the non-uniform case. In [17, Theorem 10.3], the authors provide upper and lower bounds for the collision probability in terms of a quantity they call "balance," which is the same as Rényi entropy of order two up to the base of the logarithm. "Multi-collisions" are also of interest in cryptography [18], [20]; these refer to the case where more than two inputs are mapped to the same output. In our work, the list size $L$ determines the type of collision; $L=1$ corresponds to standard collisions, while $L>1$ corresponds to multi-collisions.

\section{HYPERGRAPHS AND ZERO-ERROR LIST CODES}

A discrete channel is a triple

$$
(\mathcal{X}, W(y \mid x), \mathcal{Y})
$$

where $\mathcal{X}$ and $\mathcal{Y}$ are finite sets, and for each $x \in \mathcal{X}, W(\cdot \mid x)$ is a probability mass function on $\mathcal{Y}$. We say an output $y \in \mathcal{Y}$ is "reachable" from an input $x \in \mathcal{X}$ if $W(y \mid x)>0$.

A hypergraph $G=(\mathcal{V}, \mathcal{E})$ consists of a set of nodes $\mathcal{V}$ and a set of edges $\mathcal{E} \subseteq 2^{\mathcal{V}}$, where $2^{\mathcal{V}}$ denotes the collection of subsets of $\mathcal{V}$. We assume that $\mathcal{V}$ is finite and each edge has cardinality at least two.

The distinguishability hypergraph for a discrete channel $W$, denoted by $G(W)$, is a hypergraph with vertex set $\mathcal{X}$ and an edge set $\mathcal{E} \subseteq 2^{\mathcal{X}}$ that contains all collections of inputs that are "distinguishable" at the decoder. Formally, $\mathcal{E}$ consists of all subsets $e \subseteq \mathcal{X}$ that satisfy

$$
\forall y \in \mathcal{Y}: \prod_{x \in e} W(y \mid x)=0 ;
$$

that is, $e \subseteq \mathcal{X}$ is an edge if no $y \in \mathcal{Y}$ is reachable from all $x \in e$. Note that $G(W)$ has the property that the superset of any edge is an edge; that is, if $e \in \mathcal{E}$ and $e \subseteq e^{\prime} \subseteq \mathcal{X}$, then $e^{\prime} \in \mathcal{E}$. Proposition 1, below, shows that any hypergraph $G$ with this property is the distinguishability hypergraph of some channel $W$.

An independent set of a hypergraph $G=(\mathcal{V}, \mathcal{E})$ is a nonempty subset $\mathcal{I} \subseteq \mathcal{V}$ such that no subset of $\mathcal{I}$ is in $\mathcal{E}$.

\footnotetext{
${ }^{2}$ The attempt to find such inputs is called the birthday attack in cryptography [19. p. 187].
} 

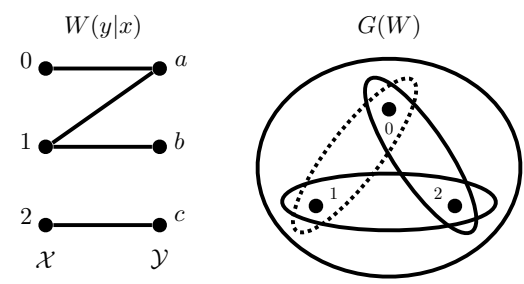

$$
\begin{aligned}
& \mathcal{X}=\{0,1,2\} \\
& \mathcal{Y}=\{a, b, c\} \\
& \mathcal{E}=\{\{0,2\},\{1,2\},\{0,1,2\}\} \\
& \mathcal{I}=\{0,1\}
\end{aligned}
$$

Fig. 1. This figure depicts the channel $(\mathcal{X}, W(y \mid x), \mathcal{Y})$ (left) and its corresponding distinguishability hypergraph $G(W)=(\mathcal{X}, \mathcal{E})$ (center). On the left, an edge connects $x \in \mathcal{X}$ and $y \in \mathcal{Y}$ if and only if $W(y \mid x)>0$. In the center, the ovals with solid lines are the edges of $G(W)$, while the oval with the dashed line represents the independent set $\mathcal{I}$.

For the channel $(\mathcal{X}, W(y \mid x), \mathcal{Y})$, an independent set of $G(W)$ corresponds to a collection of inputs $\mathcal{I} \subseteq \mathcal{X}$ for which there exists an output $y \in \mathcal{Y}$ that is reachable from any $x \in \mathcal{I}$. (See Figure 1 for a simple example.) The hypergraph $G$ is a complete multipartite $(\mathrm{CM})$ hypergraph if there exists a partition $\left\{\mathcal{I}_{j}\right\}_{j=1}^{k}$ of $\mathcal{V}$ such that each $\mathcal{I}_{j}$ is an independent set, and for every subset $e \subseteq \mathcal{V}$, either $e \in \mathcal{E}$, or $e \subseteq \mathcal{I}_{j}$ for some $1 \leq j \leq k$.

As an example, consider a deterministic channel $(\mathcal{X}, W(y \mid x), \mathcal{Y})$, where for some mapping $\varphi: \mathcal{X} \rightarrow \mathcal{Y}$,

$$
W(y \mid x)=\mathbf{1}\{y=\varphi(x)\} .
$$

For this channel, $G(W)$ is a CM hypergraph. To demonstrate this, let $\varphi(\mathcal{X})$ denote the range of $\varphi$. Then the sets $\left\{\varphi^{-1}(y)\right\}_{y \in \varphi(\mathcal{X})}$ are the independent components of $G$, where for $y \in \varphi(\mathcal{X})$,

$$
\varphi^{-1}(y):=\{x \in \mathcal{X} \mid \varphi(x)=y\} .
$$

Further, for each $e \subseteq \mathcal{V}$, either $e \subseteq \varphi^{-1}(y)$ for some $y \in \varphi(\mathcal{X})$ or $e$ contains elements from at least two independent sets, which implies $e \in \mathcal{E}$.

Proposition 1 gives a complete characterization of hypergraphs $G$ that correspond to the distinguishability hypergraphs for both general and deterministic channels.

Proposition 1. Consider a hypergraph $G=(\mathcal{V}, \mathcal{E})$. Then there exists a discrete channel $(\mathcal{X}, W(y \mid x), \mathcal{Y})$ such that $G=G(W)$ if and only if the superset of every edge of $G$ is an edge. Furthermore, there exists a deterministic channel $W$ such that $G=G(W)$ if and only if $G$ is a CM hypergraph.

Relying on the connection between channels and hypergraphs in Proposition 1. we next find a graphtheoretic condition for a mapping to be a zero-error list code. We present this condition in Proposition 2 Prior to that, we define necessary notation.

For positive integers $i$ and $j$ with $j \geq i,[i: j]$ denotes the set $\{i\}$ if $j=i$ and $\{i, i+1, \ldots, j\} j>i$. When $i=1$, we denote $[1: j]$ by $[j]$. For example, $[1]=\{1\}$ and $[2: 4]=$ $\{2,3,4\}$. For any set $\mathcal{A}$ and nonnegative integer $k \leq|\mathcal{A}|$, a $k$ subset of $\mathcal{A}$ is any set $\mathcal{B} \subseteq \mathcal{A}$ such that $|\mathcal{B}|=k$. Furthermore, the set

$$
\left(\begin{array}{l}
\mathcal{A} \\
k
\end{array}\right):=\{\mathcal{B}|\mathcal{B} \subseteq \mathcal{A},| \mathcal{B} \mid=k\},
$$

denotes the collection of all $k$-subsets of $\mathcal{A}$.
An $(M, L)$ list code for the channel $(\mathcal{X}, W(y \mid x), \mathcal{Y})$ consists of an encoder

$$
f:[M] \rightarrow \mathcal{X},
$$

and a decoder

$$
g: \mathcal{Y} \rightarrow \bigcup_{\ell=1}^{L}\left(\begin{array}{c}
{[M]} \\
\ell
\end{array}\right)
$$

The pair $(f, g)$ is an $(M, L)$ zero-error list code for channel $W$ if for every $m \in[M]$ and $y \in \mathcal{Y}$ satisfying $W(y \mid f(m))>0$, we have $m \in g(y)$.

Proposition 2 provides a necessary and sufficient condition for the existence of an $(M, L)$ zero-error list code for $W$ in terms of its distinguishability hypergraph $G(W)$.

Proposition 2. Consider a discrete channel $(\mathcal{X}, W(y \mid x), \mathcal{Y})$, positive integers $M$ and $L$, and an encoder

$$
f:[M] \rightarrow \mathcal{X} .
$$

For this encoder, a decoder

$$
g: \mathcal{Y} \rightarrow \bigcup_{\ell=1}^{L}\left(\begin{array}{c}
{[M]} \\
\ell
\end{array}\right)
$$

exists such that the pair $(f, g)$ is an $(M, L)$ zero-error list code for $W$ if and only if the image of every $(L+1)$-subset $\left\{m_{\ell}\right\}_{\ell=1}^{L+1}$ of $[M]$ under $f$ is an edge of $G(W)$.

Proposition 2 reduces the existence of an $(M, L)$ zero-error list code to the existence of an encoder with a certain property. Because of this, henceforth we say a mapping $f:[M] \rightarrow \mathcal{X}$ is an $(M, L)$ zero-error list code for the channel $W$ if it satisfies the condition stated in Proposition 2 .

For each positive integer $n$, the $n$th memoryless extension channel of $W$ is the channel

$$
\left(\mathcal{X}^{n}, W^{n}\left(y^{n} \mid x^{n}\right), \mathcal{Y}^{n}\right),
$$

where

$$
W^{n}\left(y^{n} \mid x^{n}\right):=\prod_{t \in[n]} W\left(y_{t} \mid x_{t}\right) .
$$

An $(M, L)$ zero-error list code for $W^{n}$ is referred to as an $(M, n, L)$ zero-error list code for $W$. It is possible to show that the distinguishability hypergraph of $W^{n}, G\left(W^{n}\right)$, equals $G^{n}(W)$, the $n$th co-normal power of $G(W)$ [21, Definition 2.1] which we next define.

For any positive integer $n$ and any hypergraph $G=(\mathcal{V}, \mathcal{E})$, $G^{n}=\left(\mathcal{V}_{n}, \mathcal{E}_{n}\right)$ denotes the $n$th co-normal power of $G$. The set of vertices, $\mathcal{V}_{n}$ is simply $\mathcal{V}^{n}$, and the set of edges $\mathcal{E}_{n} \subseteq \mathcal{V}^{n}$ is defined next. For each $k \geq 2$, the $k$-subset $e=\left\{v_{1}^{n}, \ldots, v_{k}^{n}\right\} \subseteq \mathcal{V}^{n}$ is in $\mathcal{E}_{n}$ if for at least one $t \in[n]$, $\left\{v_{1 t}, \ldots, v_{k t}\right\} \in \mathcal{E}$. This definition is motivated by the fact that $k$ codewords are distinguishable if and only if their components are distinguishable in at least one dimension.

In the next section, we consider randomly generated codes and present our main result. 


\section{RANDOM ZERO-ERROR LIST CODES}

Fix a sequence of probability mass functions $\left(P_{n}\left(x^{n}\right)\right)_{n=1}^{\infty}$, where $P_{n}$ is defined over $\mathcal{X}^{n}$. Our aim here is to study the performance of the sequence of random codes $F_{n}:\left[M_{n}\right] \rightarrow$ $\mathcal{X}^{n}$ over the channel $(\mathcal{X}, W(y \mid x), \mathcal{Y})$, where each $F_{n}$ is generated by $P_{n}$ in an i.i.d. manner across the messages; that is,

$$
F_{n}(1), \ldots, F_{n}\left(M_{n}\right)
$$

are $M_{n}$ i.i.d. random variables, and

$$
\forall m \in\left[M_{n}\right]: \operatorname{Pr}\left\{F_{n}(m)=x^{n}\right\}:=P_{n}\left(x^{n}\right) .
$$

We seek to find conditions on the sequence $\left(M_{n}\right)_{n=1}^{\infty}$ such that

$$
\lim _{n \rightarrow \infty} \operatorname{Pr}\left\{F_{n} \text { is an }\left(M_{n}, n, L\right) \text { zero-error list code }\right\}=1 .
$$

Theorem 4. below, provides the desired conditions. The conditions rely on a collection of functions of the pair $\left(G^{n}(W), P_{n}\right)$, denoted by

$$
\left(\theta_{L+1}^{(\ell)}\left(G^{n}(W), P_{n}\right)\right)_{\ell=1}^{L+1},
$$

which we next define.

Consider a hypergraph $G=(\mathcal{V}, \mathcal{E})$. For any positive integer $k$, let $v_{[k]}=\left(v_{1}, \ldots, v_{k}\right)$ denote an element of $\mathcal{V}^{k}$. For all $v_{[k]} \in \mathcal{V}^{k}$ and every nonempty subset $S \subseteq[k]$, define $v_{S}:=$ $\left(v_{j}\right)_{j \in S}$. Let $P$ be a probability mass function on $\mathcal{V}$ and set

$$
P\left(v_{S}\right):=\prod_{j \in S} P\left(v_{j}\right) .
$$

In addition, for each positive integer $k \geq 2$, define the mapping $\sigma_{k}: \mathcal{V}^{k} \rightarrow 2^{\mathcal{V}}$ as

$$
\sigma_{k}\left(v_{[k]}\right):=\left\{v_{1}, \ldots, v_{k}\right\} .
$$

In words, $\sigma_{k}$ maps each vector $v_{[k]} \in \mathcal{V}^{k}$ to the set containing its distinct components. For example, if $v_{[k]}=(v, \ldots, v)$ for some $v \in \mathcal{V}$, then $\sigma_{k}\left(v_{[k]}\right)=\{v\}$. When the value of $k$ is clear from context, we denote $\sigma_{k}$ with $\sigma$.

We next define functions of the pair $(G, P)$ that are instrumental in characterizing the performance of random codebooks over channels with zero-error constraints. For every positive integer $L$, define the quantity $I_{L+1}(G, P)$ as

$$
I_{L+1}(G, P):=-\frac{1}{L} \log \sum_{\substack{v_{[L+1]} \in \mathcal{V}^{L+1} \\ \sigma\left(v_{[L+1]}\right) \notin \mathcal{E}}} P\left(v_{[L+1]}\right),
$$

where $\log$ is the binary logarithm. Note that in (7), the sum

$$
\sum_{\substack{v_{[L+1]} \in \mathcal{V}^{L+1} \\ \sigma\left(v_{[L+1]}\right) \notin \mathcal{E}}} P\left(v_{[L+1]}\right)
$$

equals the probability of independently selecting $L+1$ vertices of $G$, with replacement, that are indistinguishable. The negative sign in (7) results in the nonnegativity of $I_{L+1}(G, P)$; division by $L$, as we show in Proposition 3 . makes it comparable to the Rényi entropy of order $L+1$ [22], which is defined as

$$
H_{L+1}(P):=-\frac{1}{L} \log \sum_{v \in \mathcal{V}}(P(v))^{L+1} .
$$

We also define the sequence of functions $\left(\theta_{L+1}^{(\ell)}(G, P)\right)_{\ell \in[L+1]}$. This sequence arises from the application of a second moment bound [23. Chapter 4] in proving Theorem 4 below. Set $\theta_{L+1}^{(L+1)}(G, P):=I_{L+1}(G, P)$, and for $\ell \in[L]$, let

$$
\begin{aligned}
& \theta_{L+1}^{(\ell)}(G, P):=2 I_{L+1}(G, P) \\
& +\frac{1}{L} \log \sum_{v_{[\ell]} \in \mathcal{V}^{\ell}} P\left(v_{[\ell]}\right)\left[\sum_{\substack{v_{[\ell+1: L+1]} \in \mathcal{V}^{L-\ell+1} \\
\sigma\left(v_{[L+1]}\right) \notin \mathcal{E}}} P\left(v_{[\ell+1: L+1]}\right)\right]^{2} .
\end{aligned}
$$

The sequence $\left(\theta_{L+1}^{(\ell)}(G, P)\right)_{\ell \in[L+1]}$ satisfies a number of properties which we present in Proposition 3.

Proposition 3. For every hypergraph $G=(\mathcal{V}, \mathcal{E})$, probability mass function $P$ on $\mathcal{V}$, and positive integer $L$, the following statements hold.

(i) For all $\ell \in[L+1]$,

$$
0 \leq \theta_{L+1}^{(\ell)}(G, P) \leq I_{L+1}(G, P) .
$$

(ii) We have

$$
0 \leq I_{L+1}(G, P) \leq H_{L+1}(P) .
$$

Let $\operatorname{supp}(P)$ denote the support of $P$. Then

$$
\begin{aligned}
& I_{L+1}(G, P)=0 \\
& \quad \Longleftrightarrow \forall e \subseteq \operatorname{supp}(P):(2 \leq|e| \leq L+1 \Longrightarrow e \notin \mathcal{E})
\end{aligned}
$$

and

$$
\begin{aligned}
& I_{L+1}(G, P)=H_{L+1}(P) \\
& \quad \Longleftrightarrow \forall e \subseteq \operatorname{supp}(P):(2 \leq|e| \leq L+1 \Longrightarrow e \in \mathcal{E}) .
\end{aligned}
$$

(iii) For every positive integer $n \geq 2$, define the probability mass function $P^{n}$ on $\mathcal{V}^{n}$ as

$$
\forall v^{n} \in \mathcal{V}^{n}: P^{n}\left(v^{n}\right):=\prod_{t \in[n]} P\left(v_{t}\right) .
$$

Then for all $\ell \in[L+1]$,

$$
\theta_{L+1}^{(\ell)}\left(G^{n}, P^{n}\right)=n \theta_{L+1}^{(\ell)}(G, P),
$$

where $G^{n}$ is the nth co-normal power of $G$ defined in Section [III]

For $L=1, I_{L+1}(G, P)$ has additional properties which we discuss in Appendix A.

Relying on the above notation, we next state our main result which provides upper and lower bounds on the cardinality of a randomly generated codebook that has zero error.

Theorem 4. Consider a discrete channel $(\mathcal{X}, W(y \mid x), \mathcal{Y}), a$ positive integer $L$, a sequence of probability mass functions $\left(P_{n}\left(x^{n}\right)\right)_{n=1}^{\infty}$, integers $\left(M_{n}\right)_{n=1}^{\infty}$, and random codes $\left(F_{n}\right)_{n=1}^{\infty}$. Let each $F_{n}:\left[M_{n}\right] \rightarrow \mathcal{X}^{n}$ be generated by $P_{n}$ in an i.i.d. manner across the messages. If

$$
\lim _{n \rightarrow \infty} M_{n}^{L+1} 2^{-L I_{L+1}\left(G^{n}(W), P_{n}\right)}=0,
$$


then

$$
\lim _{n \rightarrow \infty} \operatorname{Pr}\left\{F_{n} \text { is an }\left(M_{n}, n, L\right) \text { zero-error list code }\right\}=1 .
$$

Conversely, assuming (11), then for some $\ell \in[L+1]$,

$$
\lim _{n \rightarrow \infty} M_{n}^{\ell} 2^{-L \theta_{L+1}^{(\ell)}\left(G^{n}(W), P_{n}\right)}=0 .
$$

Here we provide a sketch of the proof of Theorem 4 Fix a positive integer $n$, and let $\mathcal{E}_{n} \subseteq \mathcal{X}^{n}$ denote the set of edges of $G^{n}(W)$. For every $(L+1)$-subset $S$ of $\left[M_{n}\right]$, define the random variable $Z_{S}^{(n)}$ as

$$
Z_{S}^{(n)}:=\mathbf{1}\left\{\left\{F_{n}(m)\right\}_{m \in S} \notin \mathcal{E}_{n}\right\} .
$$

In words, $Z_{S}^{(n)}$ is the indicator of the event that the codewords $\left\{F_{n}(m)\right\}_{m \in S}$ are not distinguishable. Let

$$
Z^{(n)}:=\sum_{S \in\left(\begin{array}{c}
{\left[M_{n}\right]} \\
L+1
\end{array}\right)} Z_{S}^{(n)} .
$$

Note that by Proposition 2, $F_{n}$ is an $\left(M_{n}, n, L\right)$ zero-error list code if and only if $Z^{(n)}=0$. Because of this, the remainder of the proof consists of finding lower and upper bounds for $\operatorname{Pr}\left\{Z^{(n)}=0\right\}$ and then taking the limit $n \rightarrow \infty$.

For the lower bound, we rely on Markov's inequality to show that

$$
\operatorname{Pr}\left\{Z^{(n)}=0\right\} \geq 1-\mathbb{E}\left[Z^{(n)}\right],
$$

while for the upper bound, we use the Cauchy-Schwarz inequality, which gives

$$
\operatorname{Pr}\left\{Z^{(n)}=0\right\} \leq 1-\frac{\left(\mathbb{E}\left[Z^{(n)}\right]\right)^{2}}{\mathbb{E}\left[\left(Z^{(n)}\right)^{2}\right]} .
$$

The quantities $I_{L+1}\left(G^{n}(W), P_{n}\right)$ and $\theta_{L+1}^{(\ell)}\left(G^{n}(W), P_{n}\right)$ for $\ell \in[L+1]$ appear in our calculation of $\mathbb{E}\left[Z^{(n)}\right]$ and $\mathbb{E}\left[\left(Z^{(n)}\right)^{2}\right]$, respectively. From there, 10 follows from finding sufficient conditions for $M_{n}$ such that $\mathbb{E}\left[Z^{(n)}\right] \rightarrow 0$ as $n \rightarrow \infty$ and (12) follows from finding necessary conditions on $M_{n}$ whenever

$$
\frac{\left(\mathbb{E}\left[Z^{(n)}\right]\right)^{2}}{\mathbb{E}\left[\left(Z^{(n)}\right)^{2}\right]} \rightarrow 0
$$

as $n \rightarrow \infty$. We provide the full details of this proof in Subsection V-D

In Theorem 4 if a channel $W$ and a sequence of probability mass functions $\left(P_{n}\right)_{n=1}^{\infty}$ satisfy

$$
\max _{\ell \in[L+1]} \frac{1}{\ell} \theta_{L+1}^{(\ell)}\left(G^{n}(W), P_{n}\right)=\frac{1}{L+1} I_{L+1}\left(G^{n}(W), P_{n}\right),
$$

for sufficiently large $n$, then (10), in addition to being sufficient for (11), is necessary as well. In the next corollary, we present a sufficient condition under which (15) holds. To describe this condition precisely, we require the next definition.

Consider a hypergraph $G=(\mathcal{V}, \mathcal{E})$ and a probability mass function $P$ on $\mathcal{V}$. Let $\mathcal{V}_{P}=\operatorname{supp}(P)$ and $\mathcal{E}_{P} \subseteq \mathcal{E}$ be the set of all edges whose vertices lie in $\mathcal{V}_{P}$. We refer to the hypergraph $G_{P}:=\left(\mathcal{V}_{P}, \mathcal{E}_{P}\right)$ as the subhypergraph of $G$ induced by $P$. For a fixed $n$, a sufficient condition for (15) to hold is for the subhypergraph of $G^{n}(W)$ induced by $P_{n}$ to have the CM property. (Recall the definition from Section [III) This results in the next corollary. The proof of this corollary, together with the sufficient condition for (15), appears in Subsection V-E

Corollary 5. Consider a discrete channel $(\mathcal{X}, W(y \mid x), \mathcal{Y}), a$ positive integer $L$, a sequence of probability mass functions $\left(P_{n}\left(x^{n}\right)\right)_{n=1}^{\infty}$, integers $\left(M_{n}\right)_{n=1}^{\infty}$, and random codes $\left(F_{n}\right)_{n=1}^{\infty}$. Let each $F_{n}:\left[M_{n}\right] \rightarrow \mathcal{X}^{n}$ be generated by $P_{n}$ in an i.i.d. manner across the messages. If for sufficiently large $n$, the subhypergraph of $G^{n}(W)$ induced by $P_{n}$ has the CM property, then

$$
\begin{gathered}
\lim _{n \rightarrow \infty} \operatorname{Pr}\left\{F_{n} \text { is an }\left(M_{n}, n, L\right) \text { zero-error list code }\right\}=1 \\
\Longleftrightarrow \lim _{n \rightarrow \infty} M_{n}^{L+1} 2^{-L I_{L+1}\left(G^{n}(W), P_{n}\right)}=0 .
\end{gathered}
$$

One scenario where the sufficient condition of Corollary 5 holds automatically for all $n \geq 1$ is when $G(W)$ is a CM hypergraph. Lemma 6 formally states this result.

Lemma 6. If $G=(\mathcal{V}, \mathcal{E})$ is a CM hypergraph, then the following are also $C M$ hypergraphs:

(i) The nth co-normal power of $G, G^{n}$, for all $n \geq 2$.

(ii) The induced subhypergraph of $G$ by $P, G_{P}$, for any probability mass function $P$ on $\mathcal{V}$.

When the hypergraph $G(W)$ does not have the CM property, in order to obtain a simpler version of Theorem 4, we assume that the codebook distribution is not only i.i.d. across messages, but also over the blocklength. In addition, we assume that the message set cardinality grows exponentially in the blocklength. Formally, we fix a probability mass function $P$ on $\mathcal{X}$ and a rate $R \geq 0$. Then from Theorem 4, by setting $P_{n}:=P^{n}$ and $M_{n}:=\left\lfloor 2^{n R}\right\rfloor$ for all positive integers $n$ and applying parts (i) and (iii) of Proposition 3 , we get the following corollary.

Corollary 7. Consider a discrete channel $(\mathcal{X}, W(y \mid x), \mathcal{Y})$, a positive integer $L$, probability mass function $P$ on $\mathcal{X}$, nonnegative real number $R$, and random codes $\left(F_{n}\right)_{n=1}^{\infty}$. Let each $F_{n}:\left[2^{n R}\right] \rightarrow \mathcal{X}^{n}$ be generated by $P$ in an i.i.d. manner across the messages and the blocklength. If

$$
R<\frac{L}{L+1} I_{L+1}(G, P),
$$

then

$$
\lim _{n \rightarrow \infty} \operatorname{Pr}\left\{F_{n} \text { is a }\left(2^{n R}, n, L\right) \text { zero-error list code }\right\}=1 .
$$

Conversely, if (16) holds, then

$$
R<L I_{L+1}(G, P) .
$$

Note that in Corollary 7, if $G(W)$ is a CM hypergraph, as in the next example, then using Corollary 5, the upper bound (17) can be improved to

$$
R<\frac{L}{L+1} I_{L+1}(G, P) .
$$

We next apply Corollary 5 to the noiseless channel $W=$ $(\mathcal{X}, \mathbf{1}\{y=x\}, \mathcal{X})$. Per the informal discussion in the introduction, applying our result to this channel gives the exact asymptotic behavior of the probability of coinciding birthdays 
in the birthday problem. We formalize this connection in the next corollary.

Corollary 8. Fix a positive integer $L$, a sequence of probability mass functions $\left(P_{n}\left(x^{n}\right)\right)_{n=1}^{\infty}$, integers $\left(M_{n}\right)_{n=1}^{\infty}$, and random codes $\left(F_{n}\right)_{n=1}^{\infty}$. Let each $F_{n}:\left[M_{n}\right] \rightarrow \mathcal{X}^{n}$ be generated by $P_{n}$ in an i.i.d. manner across the messages. Then

$$
\begin{aligned}
\lim _{n \rightarrow \infty} \operatorname{Pr}\left\{\exists m_{1}, \ldots, m_{L+1} \in\left[M_{n}\right]:\right. \\
\left.F_{n}\left(m_{1}\right)=\cdots=F_{n}\left(m_{L+1}\right)\right\}=0
\end{aligned}
$$

if and only if

$$
\lim _{n \rightarrow \infty} M_{n}^{L+1} 2^{-L H_{L+1}\left(P_{n}\right)}=0 .
$$

In words, to guarantee the absence of collisions of order $(L+1)$, the population size $M_{n}$ must be negligible compared to $2^{\frac{L}{L+1}} H_{L+1}\left(P_{n}\right)$.

\section{PROOFS}

In this section, we provide detailed proofs of our results.

\section{A. Proof of Proposition 1}

Proposition 1 provides necessary and sufficient conditions for a hypergraph to be the distinguishability hypergraph of a channel in both the general case and the case of deterministic channels. The necessary condition for general channels follows from our definition of the distinguishability hypergraph $G(W)$ for a channel $W$. The necessary condition for deterministic channels follows from the discussion proceeding the statement of Proposition 1 in Section III

Here we prove the sufficient condition in each case. In both cases, we start with a hypergraph $G=(\mathcal{V}, \mathcal{E})$ which has a given property. Based on $G$, we construct a discrete channel $(\mathcal{X}, W(y \mid x), \mathcal{Y})$ which gives a distinguishability hypergraph $G(W)=\left(\mathcal{V}_{W}, \mathcal{E}_{W}\right)$. Since in both cases, we set $\mathcal{X}:=\mathcal{V}$ which implies $\mathcal{V}_{W}=\mathcal{V}$, to demonstrate that $G(W)=G$, it suffices to show that $\mathcal{E}_{W}=\mathcal{E}$.

Suppose $G=(\mathcal{V}, \mathcal{E})$ is a hypergraph where the superset of every edge is an edge. Our goal is to define a discrete channel

$$
(\mathcal{X}, W(y \mid x), \mathcal{Y})
$$

such that $G=G(W)$. Set

$$
\begin{aligned}
\mathcal{X} & :=\mathcal{V} \\
\mathcal{Y} & :=2^{\mathcal{V}} \backslash \mathcal{E},
\end{aligned}
$$

and define $W$ as

$$
W(y \mid x):=\frac{1\{x \in y\}}{|\{\bar{y} \in \mathcal{Y}: x \in \bar{y}\}|} .
$$

Note that $W$ is well-defined, since for all $x \in \mathcal{X}$,

$$
\{x\} \in\{\bar{y} \in \mathcal{Y}: x \in \bar{y}\},
$$

and thus the denominator in 18 is nonzero. Following the above discussion, we next show that the set of edges of $G(W)$, $\mathcal{E}_{W}$, coincides with $\mathcal{E}$.
A nonempty $e \subseteq \mathcal{V}$ is in $\mathcal{E}_{W}$ if and only if for all $y \in \mathcal{Y}$,

$$
\prod_{x \in e} W(y \mid x)=0
$$

For a fixed $y \in \mathcal{Y},(19)$ holds if and only if

$$
\prod_{x \in e} \mathbf{1}\{x \in y\}=\mathbf{1}\{\forall x \in e: x \in y\}=\mathbf{1}\{e \subseteq y\}=0 .
$$

Therefore, a nonempty $e \subseteq \mathcal{V}$ is in $\mathcal{E}_{W}$ if and only if no superset of $e$ is in $\mathcal{Y}$, or equivalently, every superset of $e$ is in $\mathcal{E}$. Since the superset of every $e \in \mathcal{E}$ is in $\mathcal{E}$, we must have $\mathcal{E}_{W}=\mathcal{E}$.

Next assume $G=(\mathcal{V}, \mathcal{E})$ is a CM hypergraph; that is, there exists a partition $\left\{\mathcal{I}_{j}\right\}_{j=1}^{k}$ of $\mathcal{V}$ such that for each index $j$, $\mathcal{I}_{j}$ is an independent set, and for every subset $e \subseteq \mathcal{V}$, either $e \in \mathcal{E}$, or $e \subseteq \mathcal{I}_{j}$ for some $1 \leq j \leq k$. We next define a deterministic channel

$$
(\mathcal{X}, W(y \mid x), \mathcal{Y})
$$

for the hypergraph $G$ such that $G(W)=G$. Set

$$
\begin{aligned}
& \mathcal{X}:=\mathcal{V}=\bigcup_{j \in[k]} \mathcal{I}_{j} \\
& \mathcal{Y}:=[k],
\end{aligned}
$$

and define $W$ as

$$
W(y \mid x):=\mathbf{1}\left\{x \in \mathcal{I}_{y}\right\} .
$$

Note that $W$ is well-defined, since

$$
\sum_{y \in \mathcal{Y}} W(y \mid x)=\sum_{y \in \mathcal{Y}} \mathbf{1}\left\{x \in \mathcal{I}_{y}\right\}=\left|\left\{y \in \mathcal{Y}: x \in \mathcal{I}_{y}\right\}\right|=1 .
$$

Similar to the previous case, it suffices to show $\mathcal{E}_{W}$, the set of edges of $G(W)$, is the same as $\mathcal{E}$. A nonempty subset $e \subseteq \mathcal{V}$ is in $\mathcal{E}_{W}$ if and only if for all $y \in \mathcal{Y}$,

$$
\prod_{x \in e} W(y \mid x)=\prod_{x \in e} \mathbf{1}\left\{x \in \mathcal{I}_{y}\right\}=\mathbf{1}\left\{e \subseteq \mathcal{I}_{y}\right\}=0 .
$$

In words, a nonempty subset $e \subseteq \mathcal{V}$ is in $\mathcal{E}_{W}$ if and only if for all $y \in \mathcal{Y}$, $e$ is not a subset of $\mathcal{I}_{y}$, which since $G$ is a CM hypergraph with independent sets $\left\{\mathcal{I}_{y}\right\}_{y \in \mathcal{Y}}$, is equivalent to $e \in \mathcal{E}$. Thus $\mathcal{E}_{W}=\mathcal{E}$.

\section{B. Proof of Proposition 2}

This proposition provides a necessary and sufficient condition for the existence of an $(M, L)$ zero-error code over a channel in terms of its distinguishability graph.

Let $(f, g)$ be an $(M, L)$ zero-error list code for the channel $W$. If $[M]$ has a subset of cardinality $L+1$, say $\left\{m_{\ell}\right\}_{\ell=1}^{L+1}$, such that $\left\{f\left(m_{\ell}\right)\right\}_{\ell=1}^{L+1}$ is not an edge in $G(W)$, then for some $y^{*} \in \mathcal{Y}$,

$$
\prod_{\ell \in[L+1]} W\left(y^{*} \mid f\left(m_{\ell}\right)\right)>0 .
$$

For this $y^{*} \in \mathcal{Y}$ and every $\ell \in[L+1], W\left(y^{*} \mid f\left(m_{\ell}\right)\right)>0$, which implies $m_{\ell} \in g\left(y^{*}\right)$. Thus $g\left(y^{*}\right)$ contains $L+1$ or more distinct elements, which is a contradiction. 
Conversely, suppose we have an encoder $f:[M] \rightarrow \mathcal{X}$ that maps every $(L+1)$-subset of $[M]$ onto an edge of $G(W)$. For each $y \in \mathcal{Y}$, define the set

$$
\mathcal{M}_{y}:=\{m \in[M] \mid W(y \mid f(m))>0\} .
$$

Suppose for some $y^{*} \in \mathcal{Y},\left|\mathcal{M}_{y^{*}}\right|>L$. Then $\mathcal{M}_{y^{*}}$ has a subset $\mathcal{A}$ of cardinality $L+1$. By assumption, $f$ maps $\mathcal{A}$ to an edge of $G(W)$, which implies that for all $y \in \mathcal{Y}$, including $y=y^{*}$,

$$
\prod_{m \in \mathcal{A}} W(y \mid f(m))=0 .
$$

This contradicts the definition of $M_{y^{*}}$. Thus for all $y \in \mathcal{Y}$, $\left|\mathcal{M}_{y}\right| \leq L$.

Now if we define the decoder as

$$
\forall y \in \mathcal{Y}: g(y)=\mathcal{M}_{y},
$$

then the pair $(f, g)$ is an $(M, L)$ zero-error list code and the proof is complete.

\section{Proof of Proposition 3}

Proposition 3 states three key properties of the sequence of quantities $\left(\theta_{L+1}^{(\ell)}(G, P)\right)_{\ell \in[L+1]}$.

(i) We prove the nonnegativity of $\theta_{L+1}^{(\ell)}(G, P)$ first for $\ell=L+1$ and then for arbitrary $\ell \in[L]$. Recall that $\theta_{L+1}^{(L+1)}(G, P)=I_{L+1}(G, P)$. We have

$$
\begin{aligned}
\sum_{\substack{v_{[L+1]} \in \mathcal{V}^{L+1}: \\
\sigma\left(v_{[L+1]}\right) \notin \mathcal{E}}} P\left(v_{[L+1]}\right) & \leq \sum_{v_{[L+1]} \in \mathcal{V}^{L+1}} P\left(v_{[L+1]}\right) \\
& =\left(\sum_{v \in \mathcal{V}} P(v)\right)^{L+1}=1,
\end{aligned}
$$

which implies

$$
\begin{aligned}
\theta_{L+1}^{(L+1)}(G, P) & =I_{L+1}(G, P) \\
& =-\frac{1}{L} \log \sum_{\substack{v_{[L+1]} \in \mathcal{V}^{L+1}: \\
\sigma\left(v_{[L+1]}\right) \notin \mathcal{E}}} P\left(v_{[L+1]}\right) \geq 0 .
\end{aligned}
$$

For $\ell \in[L]$, we can write $\theta_{L+1}^{(\ell)}(G, P)$ as

$$
\begin{aligned}
& \theta_{L+1}^{(\ell)}(G, P)=2 I_{L+1}(G, P) \\
& +\frac{1}{L} \log \sum_{v_{[\ell]} \in \mathcal{V}^{\ell}} P\left(v_{[\ell]}\right)\left[\sum_{\substack{v_{[\ell+1: L+1]} \in \mathcal{V}^{L-\ell+1} \\
\sigma\left(v_{[L+1]}\right) \notin \mathcal{E}}} P\left(v_{[\ell+1: L+1]}\right)\right]^{2} \\
& =\frac{1}{L} \log \frac{\sum_{v_{[\ell]}} P\left(v_{[\ell]}\right)\left[\sum_{\substack{\sum_{[\ell+1: L+1]} \in \mathcal{V}^{L-\ell+1}: \\
\sigma\left(v_{[L+1]}\right) \notin \mathcal{E}}} P\left(v_{[\ell+1: L+1]}\right)\right]^{2}}{\left[\sum_{\substack{v_{[L+1]} \in \mathcal{V}^{L+1}: \\
\sigma\left(v_{[L+1]}\right) \notin \mathcal{E}}} P\left(v_{[L+1]}\right)\right]^{2}} .
\end{aligned}
$$

Note that

$$
\sum_{v_{[\ell]} \in \mathcal{V}^{\ell}} P\left(v_{[\ell]}\right)=\left(\sum_{v \in \mathcal{V}} P(v)\right)^{\ell}=1 .
$$

Therefore, by the Cauchy-Schwarz inequality,

$$
\begin{aligned}
& \sum_{v_{[\ell]} \in \mathcal{V}^{\ell}} P\left(v_{[\ell]}\right)\left[\sum_{\substack{v_{[\ell+1: L+1]} \in \mathcal{V}^{L-\ell+1} \\
\sigma\left(v_{[L+1]}\right) \notin \mathcal{E}}} P\left(v_{[\ell+1: L+1]}\right)\right]^{2} \\
& \geq\left[\sum_{\substack{v_{[\ell]} \in \mathcal{V}^{\ell} \\
\geq}} P\left(v_{[\ell]}\right) \sum_{\substack{v_{[\ell+1: L+1]} \in \mathcal{V}^{L-\ell+1}: \\
\sigma\left(v_{[L+1]}\right) \notin \mathcal{E}}} P\left(v_{[\ell+1: L+1]}\right)\right]^{2} \\
& \left.\geq \sum_{\substack{v_{[L+1]} \in \mathcal{V}^{L+1}: \\
\sigma\left(v_{[L+1]}\right) \notin \mathcal{E}}} P\left(v_{[L+1]}\right)\right]^{2},
\end{aligned}
$$

which implies $\theta_{L+1}^{(\ell)}(G, P) \geq 0$.

We next prove the upper bound on $\theta_{L+1}^{(\ell)}(G, P)$. Note that

$$
\begin{aligned}
& \sum_{v_{[\ell]} \in \mathcal{V}^{\ell}} P\left(v_{[\ell]}\right)\left[\sum_{\substack{v_{[\ell+1: L+1]} \in \mathcal{V}^{L-\ell+1} \\
\sigma\left(v_{[L+1]} \notin \mathcal{E}\right.}} P\left(v_{[\ell+1: L+1]}\right)\right]^{2} \\
\leq & \sum_{v_{[\ell]} \in \mathcal{V}^{\ell}} P\left(v_{[\ell]}\right)\left[\sum_{\substack{v_{[\ell+1: L+1]} \in \mathcal{V}^{L-\ell+1}: \\
\sigma\left(v_{[L+1]}\right) \notin \mathcal{E}}} P\left(v_{[\ell+1: L+1]}\right)\right] \\
= & \sum_{v_{[L+1]} \in \mathcal{V}^{L+1}:} P\left(v_{[L+1]}\right) \\
= & 2^{-L\left(I_{L+1}(G, P)\right.},
\end{aligned}
$$

where 21] follows from the fact that

$$
\begin{aligned}
& \quad \sum_{\substack{v_{[\ell+1: L+1]} \in \mathcal{V}^{L-\ell+1} \\
\sigma\left(v_{[L+1]} \notin \mathcal{E}\right.}} P\left(v_{[\ell+1: L+1]}\right) \\
& \leq \sum_{v_{[\ell+1: L+1]} \in \mathcal{V}^{L-\ell+1}} P\left(v_{[\ell+1: L+1]}\right) \\
& =\left(\sum_{v \in \mathcal{V}} P(v)\right)^{L-\ell+1}=1,
\end{aligned}
$$

and 22 follows from the definition of $I_{L+1}(G, P)$. Thus

$$
\theta_{L+1}^{(\ell)}(G, P) \leq 2 I_{L+1}(G, P)-I_{L+1}(G, P)=I_{L+1}(G, P) .
$$

(ii) The inequality $I_{L+1}(G, P) \geq 0$ is proved in part (i). Equality holds if and only if

$$
\forall v_{[L+1]} \in(\operatorname{supp}(P))^{L+1}: \sigma\left(v_{[L+1]}\right) \notin \mathcal{E},
$$

which is equivalent to

$$
\forall e \subseteq \operatorname{supp}(P):(2 \leq|e| \leq L+1 \Longrightarrow e \notin \mathcal{E}) .
$$

We next prove the upper bound on $I_{L+1}(G, P)$. Since each edge of $G$ has cardinality at least two, for all $v \in \mathcal{V},\{v\} \notin \mathcal{E}$. Thus

$$
\sum_{\substack{v_{[L+1]} \in \mathcal{V}^{L+1} \\ \sigma\left(v_{[L+1]}\right) \notin \mathcal{E}}} P\left(v_{[L+1]}\right) \geq \sum_{v \in \mathcal{V}}(P(v))^{L+1},
$$

which implies

$$
I_{L+1}(G, P) \leq H_{L+1}(P)
$$


where $H_{L+1}(P)$ is the Rényi entropy of order $L+1$ defined in (8). Equality holds if and only if

$$
\begin{aligned}
\forall v_{[L+1]} & \in(\operatorname{supp}(P))^{L+1}: \\
\left(v_{1}\right. & \left.=\cdots=v_{L+1}\right) \vee\left(\sigma\left(v_{[L+1]}\right) \in \mathcal{E}\right),
\end{aligned}
$$

which is equivalent to

$$
\forall e \subseteq \operatorname{supp}(P):(2 \leq|e| \leq L+1 \Longrightarrow e \in \mathcal{E}) .
$$

(iii) Fix a positive integer $n$. Let $\mathcal{E}_{n}$ denote the set of edges of $G^{n}$. Let $v_{[L+1]}^{n}$ denote the vector

$$
v_{[L+1]}^{n}:=\left(v_{1}^{n}, \ldots, v_{L+1}^{n}\right),
$$

and $\sigma_{L+1}\left(v_{[L+1]}^{n}\right)$ denote the set

$$
\sigma_{L+1}\left(v_{[L+1]}^{n}\right):=\left\{v_{1}^{n}, \ldots, v_{L+1}^{n}\right\} .
$$

Furthermore, let $\mathcal{S} \subseteq \mathcal{V}^{L+1}$ denote the set

$$
\mathcal{S}:=\left\{v_{[L+1]} \mid \sigma_{L+1}\left(v_{[L+1]}\right) \notin \mathcal{E}\right\} .
$$

Note that for each $v_{[L+1]}^{n}, \sigma_{L+1}\left(v_{[L+1]}^{n}\right) \notin \mathcal{E}_{n}$ if and only if

$$
\forall t \in[n]:\left\{v_{1 t}, \ldots, v_{(L+1) t}\right\} \notin \mathcal{E} .
$$

Thus

$$
\left\{v_{[L+1]}^{n} \mid \sigma_{L+1}\left(v_{[L+1]}^{n}\right) \notin \mathcal{E}_{n}\right\}=\mathcal{S}^{n},
$$

which implies

$$
\begin{aligned}
& \sum_{\substack{v_{[L+1]}^{n} \in\left(\mathcal{V}^{L+1}\right)^{n}: \\
\sigma_{L+1}\left(v_{[L+1]}^{n}\right) \notin \mathcal{E}_{n}}} P^{n}\left(v_{[L+1]}^{n}\right) \\
= & \sum_{v_{[L+1]}^{n} \in \mathcal{S}^{n}} P^{n}\left(v_{[L+1]}^{n}\right) \\
= & \sum_{v_{[L+1]}^{n} \in \mathcal{S}^{n}} \prod_{t \in[n]} P\left(v_{[L+1] t}\right) \\
= & \prod_{t \in[n]} \sum_{v_{[L+1] t} \in S} P\left(v_{[L+1] t}\right) \\
= & \left(\sum_{v_{[L+1]} \in \mathcal{S}} P\left(v_{[L+1]}\right)\right)^{n},
\end{aligned}
$$

where in 23), $v_{[L+1] t}=\left(v_{1 t}, \ldots, v_{(L+1) t}\right)$. Therefore,

$$
I_{L+1}\left(G^{n}, P^{n}\right)=n I_{L+1}(G, P) .
$$

Using a similar argument as above, it follows that for all $\ell \in[L]$,

$$
\theta_{L+1}^{(\ell)}\left(G^{n}, P^{n}\right)=n \theta_{L+1}^{(\ell)}(G, P) .
$$

\section{Proof of Theorem 4}

Theorem 4 provides upper and lower bounds on the size $M_{n}$ of the codebook of an $\left(M_{n}, L\right)$ zero-error list code over the sequence of channels $W^{n}\left(y^{n} \mid x^{n}\right)$ defined by (6).

We start by finding upper and lower bounds on the probability that a random mapping from $[M]$ to $\mathcal{X}$ is an $(M, L)$ zero-error list code for the channel $W{ }^{3}$ The theorem then

\footnotetext{
${ }^{3}$ Without loss of generality, we may assume that $M>L$, since if $M \leq L$, then every mapping $f:[M] \rightarrow \mathcal{X}$ is an $(M, L)$ zero-error list code.
}

follows from applying our bounds to the channel $W^{n}$ for every positive integer $n$ and taking the limit $n \rightarrow \infty$.

Consider the random mapping $F:[M] \rightarrow \mathcal{X}$, where $(F(m))_{m \in[M]}$ is a collection of i.i.d. random variables and each $F(m)$ has distribution

$$
\operatorname{Pr}\{F(m)=x\}:=P(x) .
$$

For every $S \in\left(\begin{array}{c}{[M]} \\ L+1\end{array}\right)$, define the random variable $Z_{S}$ as

$$
Z_{S}:=\mathbf{1}\left\{\{F(m)\}_{m \in S} \notin \mathcal{E}\right\} ;
$$

that is, $Z_{S}$ is the indicator of the event that $\{F(m)\}_{m \in S}$ is not an edge of the distinguishability hypergraph $G:=G(W)$. Let

$$
Z:=\sum_{S \in\left(\begin{array}{l}
{[M]} \\
L+1
\end{array}\right)} Z_{S}
$$

Note that by Proposition 2, $F$ is an $(M, L)$ zero-error list code if and only if $Z=0$. The rest of the proof consists of computing a lower and an upper bound for $\operatorname{Pr}\{Z=0\}$.

Lower Bound. By Markov's inequality,

$$
\begin{aligned}
& \operatorname{Pr}\{F \text { is an }(M, L) \text { zero-error list code }\} \\
&=\operatorname{Pr}\{Z=0\} \\
&=1-\operatorname{Pr}\{Z \geq 1\} \\
& \geq 1-\mathbb{E}[Z] .
\end{aligned}
$$

For any $S \in\left(\begin{array}{c}{[M]} \\ L+1\end{array}\right)$,

$$
\mathbb{E}\left[Z_{S}\right]=\sum_{x_{[L+1]}: \sigma\left(x_{[L+1]}\right) \notin \mathcal{E}} P\left(x_{[L+1]}\right)=2^{-L I_{L+1}(G, P)} .
$$

By linearity of expectation,

$$
\mathbb{E}[Z]=\left(\begin{array}{c}
M \\
L+1
\end{array}\right) 2^{-L I_{L+1}(G, P)} .
$$

Combining 24) and 26, gives

$$
\begin{aligned}
\operatorname{Pr}\{Z=0\} & \geq 1-\left(\begin{array}{c}
M \\
L+1
\end{array}\right) 2^{-L I_{L+1}(G, P)} \\
& \geq 1-M^{L+1} 2^{-L I_{L+1}(G, P)},
\end{aligned}
$$

where the last inequality follows from the fact that

$$
\left(\begin{array}{c}
M \\
L+1
\end{array}\right) \leq M^{L+1}
$$

Upper Bound. We apply the second moment method. By the Cauchy-Schwarz inequality,

$$
\mathbb{E}[Z]=\mathbb{E}\left[Z \mathbf{1}_{\{Z \geq 1\}}\right] \leq \sqrt{\mathbb{E}\left[Z^{2}\right] \times \operatorname{Pr}\{Z \geq 1\}},
$$

thus

$$
\operatorname{Pr}\{Z \geq 1\} \geq \frac{(\mathbb{E}[Z])^{2}}{\mathbb{E}\left[Z^{2}\right]}
$$

and therefore

$$
\operatorname{Pr}\{Z=0\} \leq 1-\frac{(\mathbb{E}[Z])^{2}}{\mathbb{E}\left[Z^{2}\right]} .
$$


To evaluate the upper bound on $\operatorname{Pr}\{Z=0\}$, we calculate $\mathbb{E}\left[Z^{2}\right]$. We have

$$
\begin{aligned}
& Z^{2}=\left[\sum_{S \in\left(\begin{array}{l}
{[M]} \\
L+1
\end{array}\right)} Z_{S}\right]^{2}
\end{aligned}
$$

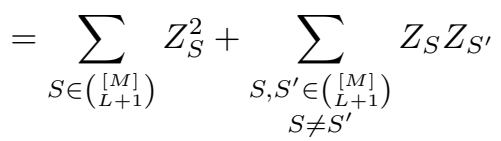

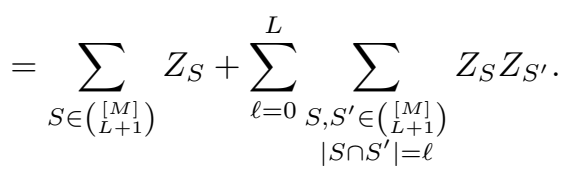

For all $\ell \in\{0,1, \ldots, L\}$, fix sets $S_{\ell}, S_{\ell}^{\prime} \in\left(\begin{array}{l}{[M]} \\ L+1\end{array}\right)$ such that $\left|S_{\ell} \cap S_{\ell}^{\prime}\right|=\ell$. When $\ell \in[L],(F(m))_{m \in S_{\ell}}$ and $(F(m))_{m \in S_{\ell}^{\prime}}$ are independent given $(F(m))_{m \in S_{\ell} \cap S_{\ell}^{\prime}}$. Thus for $\ell \in[L]$,

$$
\begin{aligned}
& \mathbb{E}\left[Z_{S_{\ell}} Z_{S_{\ell}^{\prime}}\right] \\
& \quad=\sum_{x_{[\ell]} \in \mathcal{X}^{\ell}} P\left(x_{[\ell]}\right)\left[\sum_{\substack{x_{[\ell+1: L+1]} \in \mathcal{X}^{L-\ell+1} \\
\sigma\left(x_{[L+1]}\right) \notin \mathcal{E}}} P\left(x_{[\ell+1: L+1]}\right)\right]^{2} \\
& \quad=2^{L\left(\theta_{L+1}^{(\ell)}(G, P)-2 I_{L+1}(G, P)\right)}
\end{aligned}
$$

where in 28, we use the definition of $\theta_{L+1}^{(\ell)}(G, P)$ given by 97. When $\ell=0, Z_{S_{\ell}}$ and $Z_{S_{\ell}^{\prime}}$ are independent. Thus by 25,

$$
\mathbb{E}\left[Z_{S_{0}} Z_{S_{0}^{\prime}}\right]=\left(\mathbb{E}\left[Z_{S_{0}}\right]\right)^{2}=2^{-2 L I_{L+1}(G, P)} .
$$

Equations 277, 28, and 29) together imply

$$
\begin{aligned}
& \mathbb{E}\left[Z^{2}\right]=\left(\begin{array}{c}
M \\
L+1
\end{array}\right) 2^{-L I_{L+1}(G, P)} \\
& +\left(\begin{array}{c}
M \\
0, L+1, L+1
\end{array}\right) 2^{-2 L I_{L+1}(G, P)} \\
& +\sum_{\ell=1}^{L}\left(\begin{array}{c}
M \\
\ell, L+1-\ell, L+1-\ell
\end{array}\right) 2^{L\left(\theta_{L+1}^{(\ell)}(G, P)-2 I_{L+1}(G, P)\right)}
\end{aligned}
$$

where in 30, for $\ell \in\{0,1, \ldots, L\}$, the multinomial coefficient

$$
\begin{gathered}
\left(\begin{array}{c}
M \\
\ell, L+1-\ell, L+1-\ell
\end{array}\right) \\
\quad:=\left(\begin{array}{c}
M \\
\ell
\end{array}\right)\left(\begin{array}{c}
M-\ell \\
L+1-\ell
\end{array}\right)\left(\begin{array}{c}
M-L-1 \\
L+1-\ell
\end{array}\right)
\end{gathered}
$$

equals the number of pairs $\left(S, S^{\prime}\right)$, where $S, S^{\prime} \in\left(\begin{array}{l}{[M]} \\ L+1\end{array}\right)$ and
$\left|S \cap S^{\prime}\right|=\ell$. Combining (26) with 30 now gives

$$
\begin{aligned}
\frac{\mathbb{E}\left[Z^{2}\right]}{(\mathbb{E}[Z])^{2}} & \left(\begin{array}{c}
M \\
L+1
\end{array}\right)^{-1} 2^{L I_{L+1}(G, P)}+\left(\begin{array}{c}
M \\
L+1
\end{array}\right)^{-2}\left(\begin{array}{c}
M \\
0, L+1, L+1
\end{array}\right) \\
& +\left(\begin{array}{c}
M \\
L+1
\end{array}\right)^{-2} \sum_{\ell=1}^{L}\left(\begin{array}{c}
M \\
\ell, L+1-\ell, L+1-\ell
\end{array}\right) 2^{L \theta_{L+1}^{(\ell)}(G, P)} \\
\leq & \left(\begin{array}{c}
M \\
L+1
\end{array}\right)^{-1} 2^{L I_{L+1}(G, P)}+1 \\
& +\sum_{\ell=1}^{L}\left(\begin{array}{c}
L+1 \\
\ell
\end{array}\right)^{2}\left(\begin{array}{c}
M \\
\ell
\end{array}\right)^{-1} 2^{L \theta_{L+1}^{(\ell)}(G, P)} .
\end{aligned}
$$

The inequality in 31 follows from the fact that for each $\ell \in$ $\{0,1, \ldots, L\}$,

$$
\begin{aligned}
& \left(\begin{array}{c}
M \\
L+1
\end{array}\right)^{-2}\left(\begin{array}{c}
M \\
\ell, L+1-\ell, L+1-\ell
\end{array}\right) \\
& =\left(\begin{array}{c}
L+1 \\
\ell
\end{array}\right)^{2}\left(\begin{array}{c}
M \\
\ell
\end{array}\right)^{-1} \times \frac{(M-L-1) !(M-L-1) !}{(M-\ell) !(M-2 L-2+\ell) !} \\
& =\left(\begin{array}{c}
L+1 \\
\ell
\end{array}\right)^{2}\left(\begin{array}{c}
M \\
\ell
\end{array}\right)^{-1} \prod_{j=\ell}^{L}\left(\frac{M-L-1+\ell-j}{M-j}\right) \\
& \leq\left(\begin{array}{c}
L+1 \\
\ell
\end{array}\right)^{2}\left(\begin{array}{c}
M \\
\ell
\end{array}\right)^{-1} .
\end{aligned}
$$

This completes the proof of the upper bound.

The asymptotic result, as stated in Theorem 4 follows from applying, for every $\ell \in[L+1]$, the inequality

$$
\left(\begin{array}{c}
M \\
\ell
\end{array}\right) \geq\left(\frac{M}{\ell}\right)^{\ell}
$$

\section{E. Proof of Corollary 5}

Corollary 5 describes a sufficient condition under which the upper and lower bounds in Theorem 4 coincide. To prove this corollary, we apply the next lemma to the hypergraph $G^{n}(W)$ for sufficiently large $n$.

Lemma 9. Let $G=(\mathcal{V}, \mathcal{E})$ be a hypergraph and let $P$ be a distribution on $\mathcal{V}$. If the subhypergraph $G_{P}$ induced by distribution $P$ is $C M$, then

$$
\max _{\ell \in[L+1]} \frac{1}{\ell} \theta_{L+1}^{(\ell)}(G, P)=\frac{1}{L+1} I_{L+1}(G, P) .
$$

Proof. Let $G_{P}=\left(\mathcal{V}_{P}, \mathcal{E}_{P}\right)$. Recall that $\mathcal{V}_{P}=\operatorname{supp}(P)$, which implies that for $v \in \mathcal{V} \backslash \mathcal{V}_{P}, P(v)=0$. Thus for all $\ell \in[L+1]$,

$$
\theta_{L+1}^{(\ell)}\left(G_{P}, P\right)=\theta_{L+1}^{(\ell)}(G, P) .
$$

Therefore, it suffices to prove (32) with $G$ replaced with $G_{P}$.

Since $G_{P}$ is a CM hypergraph, there exists a partition $\left\{\mathcal{I}_{j}\right\}_{j=1}^{k}$ of $\mathcal{V}_{P}$ consisting of independent sets. Define the distribution $P^{*}$ on $[k]$ as

$$
P^{*}(j):=\sum_{v \in \mathcal{I}_{j}} P(v) .
$$


In words, $P^{*}(j)$ is the weight assigned to the independent set $\mathcal{I}_{j}$ by $P$. Since $G_{P}$ is a CM hypergraph, $\sigma\left(v_{[L+1]}\right) \notin \mathcal{E}$ if and only if there exists some $j$ such that $\sigma\left(v_{[L+1]}\right) \subseteq \mathcal{I}_{j}$. Thus

$$
\begin{aligned}
& I_{L+1}\left(G_{P}, P\right) \\
& =-\frac{1}{L} \log \sum_{v_{[L+1]} \in \mathcal{V}^{L+1}: \sigma\left(v_{[L+1]}\right) \notin \mathcal{E}} P\left(v_{[L+1]}\right) \\
& =-\frac{1}{L} \log \sum_{j=1}^{k} \sum_{v_{[L+1]} \in \mathcal{V}^{L+1}: \sigma\left(v_{[L+1]}\right) \subseteq \mathcal{I}_{j}} P\left(v_{[L+1]}\right) \\
& =-\frac{1}{L} \log \sum_{j=1}^{k}\left(P^{*}(j)\right)^{L+1} \\
& =H_{L+1}\left(P^{*}\right) \text {, }
\end{aligned}
$$

where $H_{L+1}$ denotes the Rényi entropy of order $L+1$. Similarly, for all $\ell \in[L+1]$ we have

$$
\theta_{L+1}^{(\ell)}\left(G_{P}, P\right)=2 H_{L+1}\left(P^{*}\right)-\frac{2 L+1-\ell}{L} H_{2 L+2-\ell}\left(P^{*}\right) .
$$

Using (33), we see that proving (32) is equivalent to showing that for all $\ell \in[L+1]$,

$$
\left(\sum_{j=1}^{k} P^{*}(j)^{2 L+2-\ell}\right)^{\frac{1}{2 L+2-\ell}} \leq\left(\sum_{j=1}^{k} P^{*}(j)^{L+1}\right)^{\frac{1}{L+1}},
$$

which follows from the well-known fact that for all $p \geq q$, the $q$-norm dominates the $p$-norm.

\section{F. Proof of Lemma 6}

For both parts, let $G=(\mathcal{V}, \mathcal{E})$ be a $\mathrm{CM}$ hypergraph. By definition, there exists a collection of independent sets $\left\{\mathcal{I}_{k}\right\}_{k=1}^{K}$ that is a partition of $\mathcal{V}$ and for every $e \subseteq \mathcal{V}$, either $e \in \mathcal{E}$ or $e \subseteq \mathcal{I}_{k}$ for some $k \in[K]$.

In part (i), we demonstrate that for any $n \geq 2, G^{n}=$ $\left(\mathcal{V}^{n}, \mathcal{E}_{n}\right)$ is a CM hypergraph. First note that for any $n \geq 2$, we can write $\mathcal{V}^{n}$, the set of vertices of $G^{n}$, as

$$
\mathcal{V}^{n}=\bigcup_{k_{1}, \ldots, k_{n} \in[K]} \mathcal{I}_{k_{1}} \times \cdots \times \mathcal{I}_{k_{n}} .
$$

We next show that an arbitrary subset of $\mathcal{V}^{n}$, say $\left\{v_{1}^{n}, \ldots, v_{\ell}^{n}\right\}$, is in $\mathcal{E}_{n}$ if and only if

$$
\forall k_{1}, \ldots, k_{n} \in[K]:\left\{v_{1}^{n}, \ldots, v_{\ell}^{n}\right\} \nsubseteq \mathcal{I}_{k_{1}} \times \cdots \times \mathcal{I}_{k_{n}} .
$$

By definition, $\left\{v_{1}^{n}, \ldots, v_{\ell}^{n}\right\}$ is an edge in $G^{n}$ if and only if for some $t \in[n],\left\{v_{1 t}, \ldots, v_{\ell t}\right\}$ is an edge in $G$. Since $G$ is a $\mathrm{CM}$ hypergraph, the latter condition holds if and only if

$$
\exists t \in[n] \text { such that } \forall j \in[k]:\left\{v_{1 t}, \ldots, v_{\ell t}\right\} \nsubseteq \mathcal{I}_{j},
$$

which is equivalent to (34).

In part (ii), we demonstrate that the $\mathrm{CM}$ property is inherited by subhypergraphs of $G$ induced by probability mass functions. In particular, we show that for any probability mass function $P$ defined on $\mathcal{V}, G_{P}=\left(\mathcal{V}_{P}, \mathcal{E}_{P}\right)$ is a CM hypergraph.

To this end, we need to exhibit a partition $\left\{\mathcal{J}_{\ell}\right\}_{\ell=1}^{L}$ of $\mathcal{V}_{P}$ such that each $\mathcal{J}_{\ell}$ is an independent set of $G_{P}$ and for every $e \subseteq \mathcal{V}_{P}$, either $e \in \mathcal{E}_{P}$ or $e \subseteq \mathcal{I}_{\ell}$ for some $\ell \in[L]$. First, for every $\ell \in[K]$, define

$$
\mathcal{J}_{\ell}:=\mathcal{I}_{\ell} \cap \operatorname{supp}(P) .
$$

Let $\mathcal{A}$ be a subset of $\mathcal{J}_{\ell}$. Then $\mathcal{A}$ is a subset of $\mathcal{I}_{\ell}$ as well, and since the $\mathcal{I}_{\ell}$ is an independent set of $G, \mathcal{A} \notin \mathcal{E}$. Thus

$$
\mathcal{A} \notin \mathcal{E} \cap \operatorname{supp}(P)=\mathcal{E}_{P} .
$$

Therefore, every $\mathcal{J}_{\ell}$ is either empty or an independent set of $G_{P}$. Now for some $\mathcal{L} \subseteq[K]$, let $\left\{\mathcal{J}_{\ell}\right\}_{\ell \in \mathcal{L}}$ denote exactly those $\mathcal{J}_{\ell}$ sets that are nonempty. Then we have

$$
\begin{aligned}
\bigcup_{\ell \in \mathcal{L}} J_{\ell} & =\bigcup_{\ell \in \mathcal{L}}\left(I_{\ell} \cap \operatorname{supp}(P)\right) \\
& =\left(\bigcup_{\ell \in \mathcal{L}} I_{\ell}\right) \cap \operatorname{supp}(P) \\
& =\mathcal{V} \cap \operatorname{supp}(P)=\mathcal{V}_{P} .
\end{aligned}
$$

Thus $\left\{\mathcal{J}_{\ell}\right\}_{\ell \in \mathcal{L}}$ is a partition of $\mathcal{V}_{P}$.

Next, to establish the CM property of $G_{P}$, consider a nonempty $e \subseteq \mathcal{V}_{P}$. Then $e \subseteq \mathcal{V}$. Since $G$ is a CM hypergraph, either $e \in \mathcal{E}$ or for some $\ell \in[K], e \subseteq \mathcal{I}_{\ell}$. If $e \in \mathcal{E}$, since $e$ is also a subset of $\mathcal{V}_{P}$, by definition, $e \in \mathcal{E}_{P}$. On the other hand, if for some $\ell \in[K], e \subseteq \mathcal{I}_{\ell}, e \subseteq \mathcal{V}_{P}$ implies $e \subseteq \mathcal{J}_{\ell}$. Thus $G_{P}$ is a CM hypergraph as well.

\section{G. Proof of Corollary 8}

This corollary provides a necessary and sufficient condition for a randomly generated codebook of size $M_{n}$ to have at most $L+1$ identical codewords with probability converging to one as $n$ grows without bound.

Let $W=(\mathcal{X}, 1\{y=x\}, \mathcal{X})$ denote the noiseless channel on $\mathcal{X}$. Then every $e \subseteq \mathcal{X}$ with $|e| \geq 2$ is an edge of $G(W)$. Similarly, for any $n \geq 2$ and any $e \subset \mathcal{X}^{n}$ with $|e| \geq 2$, $e$ is an edge of $G^{n}(W)$. Therefore, for distinct messages $m_{1}, \ldots, m_{L+1} \in\left[M_{n}\right]$, we have $F_{n}\left(m_{1}\right)=\cdots=F_{n}\left(m_{L+1}\right)$ if and only if

$$
\left(F_{n}\left(m_{1}\right), \ldots, F_{n}\left(m_{L+1}\right)\right) \text { is not an edge in } G^{n}(W) .
$$

Now Proposition 2 implies that the equality

$$
\begin{aligned}
\lim _{n \rightarrow \infty} \operatorname{Pr}\{ & \exists m_{1}, \ldots, m_{L+1} \in\left[M_{n}\right]: \\
& \left.F_{n}\left(m_{1}\right)=\cdots=F_{n}\left(m_{L+1}\right)\right\}=0
\end{aligned}
$$

holds if and only if

$$
\lim _{n \rightarrow \infty} \operatorname{Pr}\left\{F_{n} \text { is an }\left(M_{n}, n, L\right) \text { zero-error list code }\right\}=1 .
$$

Since $G^{n}(W)$ is a $\mathrm{CM}$ hypergraph for any $n \geq 2$, by Lemma 6 (ii), so is its induced hypergraph by $P_{n}$. Therefore, we can apply Corollary 5 and conclude that 35 holds if and only if

$$
\lim _{n \rightarrow \infty} M_{n}^{L+1} 2^{-L H_{L+1}\left(P_{n}\right)}=0 .
$$




\section{CONCLUSION}

Our work here lies in the intersection of information theory and combinatorics as we use random coding and tools from graph theory to obtain a deeper understanding of the zeroerror list decoding problem. We derive upper and lower bounds on the rate of a randomly generated codebook as a function of the channel and the distribution according to which the codebook is generated. In addition, we can view this work as an extension of the birthday problem to noisy channels, which we obtain by reformulating the birthday problem as a channel coding problem using randomly generated codebooks.

\section{APPENDIX A}

\section{Properties OF $I_{2}(G, P)$}

In this appendix, we describe two properties of $I_{2}(G, P)$. In the first part, we state the Motzkin-Straus theorem [24], which gives the maximum of $I_{2}(G, P)$ over all distributions $P$ for a fixed graph $G$. In the second part, we show that $I_{2}(G, P)$ is always less than or equal to Körner's graph entropy [25]. Prior to that however, we demonstrate that when studying the quantity $I_{2}(G, P)$, it suffices to limit $G$ to graphs (that is, hypergraphs where all edges have cardinality two).

Consider a hypergraph $G=(\mathcal{V}, \mathcal{E})$ and a probability mass function $P$ defined on $\mathcal{V}$. First, in $(7)$, if we let $L=1$, we get

$$
I_{2}(G, P)=-\frac{1}{2} \log \sum_{\substack{v_{1}, v_{2} \in \mathcal{V} \\\left\{v_{1}, v_{2}\right\} \notin \mathcal{E}}} P\left(v_{1}\right) P\left(v_{2}\right) .
$$

From (37) it is clear that $I_{2}(G, P)$ only depends on subsets of $\mathcal{E}$ that have cardinality two. Thus we limit our discussion here to graphs without loss of generality.

\section{A. The Motzkin-Straus Theorem}

Consider a graph $G=(\mathcal{V}, \mathcal{E})$. Motzkin and Straus [24] prove that

$$
\max _{P} I_{2}(G, P)=\log \omega(G),
$$

where the maximum is over all distributions $P$ defined on $\mathcal{V}$, and $\omega(G)$ is the cardinality of the largest clique in $G$. An implication of this result is Turán's graph theorem [26], which states that

$$
\omega(G) \geq \frac{|\mathcal{V}|^{2}}{|\mathcal{V}|^{2}-2|\mathcal{E}|} .
$$

To see this, let $P$ be the uniform distribution on $\mathcal{V}$. Then

$$
I_{2}(G, P)=-\log \sum_{v, v^{\prime}:\left\{v, v^{\prime}\right\} \notin \mathcal{E}} \frac{1}{|\mathcal{V}|^{2}}=\log \frac{|\mathcal{V}|^{2}}{|\mathcal{V}|^{2}-2|\mathcal{E}|},
$$

and (38) follows by the Motzkin-Straus theorem. We remark that extensions of the Motzkin-Straus theorem to hypergraphs appear in [27]-[30].

While the Motzkin-Straus theorem is concerned with the maximum of $I_{2}(G, P)$ over all distributions $P$ for a fixed graph $G$, we can also determine the maximum of $I_{2}(G, P)$ over all graphs $G$ for a fixed probability distribution $P$. From Proposition 3. Part (ii) it follows that for any distribution $P$ defined on a set $\mathcal{V}$,

$$
\max _{G} I_{2}(G, P)=H_{2}(P),
$$

where the maximum is over all graphs $G=(\mathcal{V}, \mathcal{E})$. In 39p, the maximum is achieved when $G$ is the complete graph on the support of $P$.

\section{B. Relation with Körner's Graph Entropy}

Consider a graph $G$ with vertex set $\mathcal{X}$. Let $P$ be a probability distribution on $\mathcal{X}$ and $\mathcal{Y} \subseteq 2^{\mathcal{X}}$ be the set of all independent subgraphs of $G$. Let $\Delta(G, P)$ denote the set of all probability distributions $P(x, y)$ on $\mathcal{X} \times \mathcal{Y}$ whose marginal on $\mathcal{X}$ equals $P(x)$, and

$$
\operatorname{Pr}\{X \in Y\}=\sum_{(x, y): x \in y} P(x, y)=1 .
$$

For the graph $G$ and probability distribution $P$, Körner's graph entropy [25] is defined by

$$
H_{1}(G, P)=\min _{\Delta(G, P)} I(X ; Y) .
$$

Our aim is to define $H_{2}(G, P)$ in a similar manner to how $H_{2}(P)$, the Rényi entropy of order 2, is defined. One way to accomplish this task is through the use of Jensen's inequality. Applying Jensen's inequality to Shannon entropy gives

$$
\begin{aligned}
H_{1}(P) & =-\sum_{x} P(x) \log P(x) \\
& \geq-\log \left(\sum_{x}(P(x))^{2}\right)=H_{2}(P) .
\end{aligned}
$$

Analogously, applying Jensen's inequality to the mutual information in (40) gives

$$
\begin{aligned}
I(X ; Y) & =-\sum_{(x, y): x \in y} P(x, y) \log \frac{P(x) P(y)}{P(x, y)} \\
& \geq-\log \left(\sum_{(x, y): x \in y} P(x) P(y)\right),
\end{aligned}
$$

Thus we define $H_{2}(G, P)$ as

$$
\begin{aligned}
H_{2}(G, P) & =\min _{\Delta(G, P)}-\log \left(\sum_{(x, y): x \in y} P(x) P(y)\right) \\
& \leq H_{1}(G, P) .
\end{aligned}
$$

Our next proposition relates $H_{2}(G, P)$ and $I_{2}(G, P)$ from which it follows that $I_{2}(G, P) \leq H_{1}(G, P)$.

Proposition 10. For any graph $G$ and any probability distribution $P$ defined on its vertices, $I_{2}(G, P) \leq H_{2}(G, P)$.

Proof. Let $P(x, y) \in \Delta(G, P)$. Then

$$
\sum_{x \in y} P(x) P(y)=\sum_{x, x^{\prime}} P(x) P\left(x^{\prime}\right) \sum_{y} P\left(y \mid x^{\prime}\right) \mathbf{1}\left\{x, x^{\prime} \in y\right\} .
$$

Since every $y$ is an independent subgraph of $G$, if $x, x^{\prime} \in y$, then $\left(x, x^{\prime}\right) \notin \mathcal{E}$. Thus

$$
\mathbf{1}\left\{x, x^{\prime} \in y\right\} \leq \mathbf{1}\left\{\left(x, x^{\prime}\right) \notin \mathcal{E}\right\},
$$

which implies

$$
\sum_{y} P\left(y \mid x^{\prime}\right) \mathbf{1}\left\{x, x^{\prime} \in y\right\} \leq \mathbf{1}\left\{\left(x, x^{\prime}\right) \notin \mathcal{E}\right\} .
$$


By (41), we have

$$
\sum_{x \in y} P(x) P(y) \leq \sum_{x, x^{\prime}} P(x) P\left(x^{\prime}\right) \mathbf{1}\left\{\left(x, x^{\prime}\right) \notin \mathcal{E}\right\} .
$$

Calculating the logarithm of both sides and maximizing the left hand side over $\Delta(G, P)$ gives $H_{2}(G, P) \geq I_{2}(G, P)$.

\section{APPENDIX B}

\section{CONNECTION TO RÉNYI'S RESULT}

We next state Rényi's result [13, Equation (5.3)] in the context of zero-error list codes over the noiseless channel. For the noiseless channel setting defined by (3), fix $\epsilon \in(0,1)$, positive integer $L \geq 1$, and probability mass function $P$ on $\mathcal{X}$. For every positive integer $n$ and $x^{n} \in \mathcal{X}^{n}$, define

$$
P_{n}\left(x^{n}\right):=\prod_{i=1}^{n} P\left(x_{i}\right) .
$$

For positive integers $M$ and $n$, let $\Phi_{M, n}:[M] \rightarrow \mathcal{X}^{n}$ be a random mapping with i.i.d. values $\Phi_{M, n}(1), \ldots, \Phi_{M, n}(M)$, each with distribution $P_{n}\left(x^{n}\right)$. Define $n_{L+1}^{*}(M, \epsilon)$ as the least positive integer $n$ for which

$$
\operatorname{Pr}\left\{\Phi_{M, n} \text { is an }(M, n, L) \text { zero-error list code }\right\} \geq 1-\epsilon .
$$

In [13], Rényi states that

$$
\lim _{M \rightarrow \infty} \frac{\log M}{n_{L+1}^{*}(M, \epsilon)}=\frac{L}{L+1} \cdot H_{L+1}(P) .
$$

We next describe a connection between Rényi's result and our result concerning the birthday problem. Consider the scenario where the distribution of each codeword is given by (42), the size of the message set equals $M_{n}:=\left\lfloor 2^{n R}\right\rfloor$ for some $R \geq 0$, and (35) holds. We show that both (36) and (43) imply $\sqrt{4}$

$$
R \leq \frac{L}{L+1} \cdot H_{L+1}(P) .
$$

First note that for $P_{n}$ given by (42), (36) together with Proposition 3 (iii), gives

$$
\lim _{n \rightarrow \infty}\left[(L+1) \log \left\lfloor 2^{n R}\right\rfloor-n L H_{L+1}(P)\right]=-\infty,
$$

which directly leads to (44).

Next we show that (43) implies (44). First note that for all $n, F_{n}$ has the same distribution as $\Phi_{M_{n}, n}$. In addition, by assumption, for sufficiently large $n$,

$$
\operatorname{Pr}\left\{F_{n} \text { is an }\left(M_{n}, n, L\right) \text { zero-error list code }\right\} \geq 1-\epsilon .
$$

Therefore, by the definition of $n_{L+1}^{*}$, we have

$$
n \geq n_{L+1}^{*}\left(M_{n}, \epsilon\right) .
$$

Thus if $R>0$,

$$
\begin{aligned}
\lim _{M \rightarrow \infty} \frac{\log M}{n_{L+1}^{*}(M, \epsilon)} & =\lim _{n \rightarrow \infty} \frac{\log \left\lfloor 2^{n R}\right\rfloor}{n_{L+1}^{*}\left(\left\lfloor 2^{n R}\right\rfloor, \epsilon\right)} \\
& \geq \lim _{n \rightarrow \infty} \frac{1}{n} \log \left\lfloor 2^{n R}\right\rfloor=R .
\end{aligned}
$$

Applying (43) completes the proof.

\footnotetext{
${ }^{4}$ More precisely, 36 gives 44 with strict inequality.
}

\section{ACKNOWLEDGMENT}

The first author thanks Ming Fai Wong for helpful discussions regarding an earlier version of Theorem 4

\section{REFERENCES}

[1] C. E. Shannon, "The zero error capacity of a noisy channel," IRE Trans Inf. Theory, vol. 2, no. 3, pp. 8-19, 1956

[2] - "A mathematical theory of communication," Bell Syst. Tech. J., vol. 27, pp. 379-423, 623-656, 1948.

[3] T. M. Cover and J. A. Thomas, Elements of Information Theory, 2nd ed. John Wiley \& Sons, Inc., Hoboken, New Jersey, 2006.

[4] P. Elias, "Zero error capacity under list decoding," IEEE Trans. Inf. Theory, vol. 34, no. 5, pp. 1070-1074, 1988.

[5] — , "Error-correcting codes for list decoding," IEEE Trans. Inf. Theory, vol. 37, no. 1, pp. 5-12, 1991.

[6] J. Körner and K. Marton, "On the capacity of uniform hypergraphs," IEEE Trans. Inf. Theory, vol. 36, no. 1, pp. 153-156, 1990.

[7] M. H. Gail, G. H. Weiss, N. Mantel, and S. J. O'Brien, "A solution to the generalized birthday problem with application to allozyme screening for cell culture contamination," J. Appl. Prob., vol. 16, no. 2, pp. 242-251, 1979.

[8] T. S. Nunnikhoven, "A birthday problem solution for nonuniform birth frequencies," Am. Stat., vol. 46, no. 4, pp. 270-274, 1992.

[9] C. Stein, "Application of Newton's identities to a generalized birthday problem and to the Poisson binomial distribution," Stanford University - Department of Statistics, Tech. Rep. 354, September 1990

[10] J. Körner and A. Orlitsky, "Zero-error information theory," IEEE Trans. Inf. Theory, vol. 44, no. 6, pp. 2207-2229, 1998.

[11] E. Arıkan, "An upper bound on the zero-error list-coding capacity," IEEE Trans. Inf. Theory, vol. 40, no. 4, pp. 1237-1240, 1994.

[12] İ. E. Telatar, "Zero-error list capacities of discrete memoryless channels," IEEE Trans. Inf. Theory, vol. 43, no. 6, pp. 1977-1982, 1997.

[13] A. Rényi, "On the foundations of information theory," Rev. Int. Statist. Inst., vol. 33, no. 1, pp. 1-14, 1965.

[14] A. Fujiwara, "Quantum birthday problems: Geometrical aspects of quantum random coding," IEEE Trans. Inf. Theory, vol. 47, no. 6, pp. 2644-2649, 2001

[15] R. Arratia, L. Goldstein, and L. Gordon, "Two moments suffice for Poisson approximations: The Chen-Stein method," Ann. Prob., vol. 17, no. 1 , pp. 9-25, 1989.

[16] — "Poisson approximation and the Chen-Stein method," Stat. Sci., vol. 5 , no. 4, pp. 403-434, 1990.

[17] M. Bellare and T. Kohno, "Hash function balance and its impact on birthday attacks," in Advances in Cryptology - EUROCRYPT 2004, C. Cachin and J. Camenisch, Eds. Springer-Verlag, Berlin/Heidelberg, Germany, 2004

[18] A. Joux, "Multicollisions in iterated hash functions. Application to cascaded constructions," in Advances in Cryptology - CRYPTO 2004, M. Franklin, Ed. Springer, Germany, 2004.

[19] —, Algorithmic Cryptanalysis, 1st ed. Chapman \& Hall/CRC, Boca Raton, FL, 2009.

[20] K. Suzuki, D. Tonien, K. Kurosawa, and K. Toyota, "Birthday paradox for multi-collisions," in Information Security and Cryptology - ICISC 2006, M. S. Rhee and B. Lee, Eds. Springer-Verlag, Berlin/Heidelberg, Germany, 2006

[21] G. Simonyi, "Graph entropy: A survey," in DIMACS Series in Discrete Mathematics and Theoretical Computer Science, vol. 20. American Mathematical Society, Providence, Rhode Island, 1995, pp. 399-441.

[22] A. Rényi, "On measures of information and probability," in Proc. Fourth Berkeley Symp. on Math. Statist. and Prob., vol. 1. University of California Press, 1961, pp. 547-561.

[23] N. Alon and J. H. Spencer, The Probabilistic Method, 4th ed. John Wiley \& Sons, Inc., Hoboken, New Jersey, 2015.

[24] T. S. Motzkin and E. G. Straus, "Maxima for graphs and a new proof of a theorem of Turán," Canad. J. Math., vol. 17, pp. 533-540, 1965.

[25] J. Körner, "Coding of an information source having ambiguous alphabet and the entropy of graphs," in Trans. 6th Prague Conf. Information Theory. Academia, 1973, pp. 411-425.

[26] M. Aigner, "Turán's graph theorem," Am. Math. Monthly, vol. 102, no. 9, pp. 808-816, 1995

[27] V. T. Sós and E. G. Straus, "Extremals of functions on graphs with applications to graphs and hypergraphs," J. Combin. Theory Ser. B, vol. 32 , no. 3 , pp. 246-257, 1982. 
[28] P. Frankl and V. Rödl, "Hypergraphs do not jump," Combinatorica, vol. 4, no. 2-3, pp. 149-159, 1984

[29] S. R. Bulò and M. Pelillo, "A generalization of the Motzkin-Straus theorem to hypergraphs," Optim. Lett., vol. 3, no. 2, pp. 287-295, 2009.

[30] Y. Peng, H. Peng, Q. Tang, and C. Zhao, "An extension of the MotzkinStraus theorem to non-uniform hypergraphs and its applications," Discrete Appl. Math., vol. 200, pp. 170-175, 2016.

Parham Noorzad (S'11-M'17) received the B.Sc. degree in electrical engineering from the University of Tehran in 2012 and the M.Sc. and Ph.D degrees in electrical engineering from the California Institute of Technology in 2013 and 2017, respectively. His research interests include machine learning, wireless communications, and information theory.

Michelle Effros (S'93-M'95-SM'03-F'09) received the B.S. (Hons.), M.S. and $\mathrm{Ph} . \mathrm{D}$. degrees in electrical engineering from Stanford University, in 1989, 1990, and 1994, respectively.

She joined the faculty at the California Institute of Technology in 1994, where she is currently the George Van Osdol Professor of Electrical Engineering. Her research interests include information theory, network coding, data compression, and communications. She is a member of Tau Beta Pi, Phi Beta Kappa, and Sigma Xi. She was a member of the Advisory Committee and the Committee of Visitors for the Computer and Information Science and Engineering (CISE) Directorate at the National Science Foundation from 2009 to 2012 and in 2014, respectively. She received the Stanford's Frederick Emmons Terman Engineering Scholastic Award (for excellence in engineering) in 1989, the Hughes Masters Full-Study Fellowship in 1989, the National Science Foundation Graduate Fellowship in 1990, the AT\&T Ph.D. Scholarship in 1993, the NSF CAREER Award in 1995, the Charles Lee Powell Foundation Award in 1997, the Richard Feynman-Hughes Fellowship in 1997, and an Okawa Research Grant in 2000. She was cited by Technology Review as one of the world's top young innovators in 2002 She and her coauthors received the Communications Society and Information Theory Society Joint Paper Award in 2009. She served as an Editor of the IEEE Information Theory Society Newsletter from 1995 to 1998 and as a member of the Board of Governors of the IEEE Information Theory Society from 1998 to 2003 and from 2008 to 2017, serving in the role of President of the Information Theory Society in 2015. She served on the IEEE Signal Processing Society Image and Multi-Dimensional Signal Processing (IMDSP) Technical Committee from 2001 to 2007 and on ISAT from 2006 to 2009. She served as an Associate Editor for the 2006 joint Special Issue on Networking and Information Theory in the IEEE Transactions on Information Theory and the IEEE Transactions on Networking/ACM Transactions on Networking and as an Associate Editor for Source Coding for the IEEE Transactions on Information Theory from 2004 to 2007. She has served on numerous technical program committees and review boards, including serving as the General Co-Chair for the 2009 Network Coding Workshop and the Technical Program Committee Co-Chair for the 2012 IEEE International Symposium on Information Theory.

Michael Langberg (M'07-SM'15) received his B.Sc. in mathematics an computer science from Tel-Aviv University in 1996, and his M.Sc. and Ph.D. in computer science from the Weizmann Institute of Science in 1998 and 2003 respectively. Between 2003 and 2006, he was a postdoctoral scholar in the Electrical Engineering and Computer Science departments at the California Institute of Technology, and between 2007 and 2012 he was in the Department of Mathematics and Computer Science at The Open University of Israel. Prof. Langberg is currently a professor in the Department of Electrical Engineering at the University at Buffalo.
Prof. Langberg's research addresses the mathematical foundations of information, and in particular the design and analysis of efficient and reliable algorithms for the communication, management, and storage of information. Major topics of study include the fundamental algorithmic and combinatorial challenges that arise in the study of point-to-point communication, network communication, data storage, and succinct data representation. Prof. Langberg was an Associate Editor for the IEEE Transactions on Information Theory during the years 2012-2015 and the Editor of the IEEE Information Theory Society Newsletter during the years 2015-2018.
Victoria Kostina (S'12-M'14) received a bachelor's degree from Moscow Institute of Physics and Technology (2004), where she was affiliated with the Institute for Information Transmission Problems of the Russian Academy of Sciences, a master's degree from University of Ottawa (2006), and a $\mathrm{PhD}$ from Princeton University (2013). She joined Caltech in the fall of 2014, where she is currently a Professor of Electrical Engineering and Computing and Mathematical Sciences. She received the Natural Sciences and Engineering Research Council of Canada postgraduate scholarship (20092012), the Princeton Electrical Engineering Best Dissertation Award (2013), the Simons-Berkeley research fellowship (2015) and the NSF CAREER award (2017). Kostina's research spans information theory, coding, control, learning, and communications. 